\title{
Experimental assessment of the extent of orientational short-range order in liquids
}

\author{
L. C. Pardo, ${ }^{1}$ N. Veglio, ${ }^{2}$ F. J. Bermejo, ${ }^{2}$ and J. Ll. Tamarit ${ }^{1}$ \\ ${ }^{1}$ Department Física i Enginyeria Nuclear, E.T.S.E.I.B., Universitat Politècnica de Catalunya, Diagonal 647, 08028 Barcelona, Catalonia, \\ Spain \\ ${ }^{2}$ CSIC-Department Electricity and Electronics, UPV/EHU, Box 644, 4880 Bilbao, Spain
}

\author{
G. J. Cuello \\ Institut Laue Langevin, 6 Rue Jules Horowitz, Boîte Postal 156x, F-38042 Grenoble Cedex 9, France \\ (Received 9 December 2004; revised manuscript received 21 March 2005; published 25 July 2005)
}

\begin{abstract}
The spatial extent of orientational short-range order in liquid and rotationally disordered phases of two halogen-substituted methanes are examined by means of neutron diffraction. The pair distribution functions measured within the liquids are compared to those corresponding to the rotator-phase crytals where the separation between orientational and positional correlations is enabled by the presence of time-averaged fcc lattices. The two condensed systems under scrutiny comprise a material devoid $\left(\mathrm{CCl}_{4}\right)$ and another $\left[\mathrm{C}\left(\mathrm{CD}_{3}\right)_{3} \mathrm{Cl}\right]$ having a relatively strong molecular dipole moment. Our results show orientational correlations to be confined below the second coordination sphere. Furthermore, contrary to what could be expected, angular correlations within the nonpolar material are of comparable strength to those present within the polar $\mathrm{C}\left(\mathrm{CD}_{3}\right)_{3} \mathrm{Cl}$.
\end{abstract}

DOI: $10.1103 /$ PhysRevB.72.014206

PACS number(s): 61.43.-j, 61.20.-p, 61.72.-y

\section{INTRODUCTION}

In the past few years research on the microscopic structure and dynamics of rotationally or orientationally disordered (OD) crystals has attracted renewed attention. ${ }^{1}$ The underlying reason beneath such an effort stems from the close behaviors shown by these systems to those exhibited by structurally disordered matter. In fact, several studies ${ }^{2,3}$ have reported on the proximity of dynamic behaviors shown by materials in their liquid and amorphous states to those found in the corresponding rotator-phase or orientationally disordered states.

In contrast to such progress within the realm of dynamical aspects, the advance in our knowledge of details concerning the microscopic structure of orientationally disordered crystals has been far more modest. Interest on such a topic stems from a wide variety of fields stemming from condensed molecular bodies ${ }^{4}$ to polymers and other large-scale aggregates ${ }^{5}$ or imperfectly ordered arrays such as stripe patterns. ${ }^{6}$

Within the field of interest, emphasis is most of the times focused on the relationship between the time-averaged crystal structures to that shown by the same material within its liquid or glassy phases. The driving force behind such efforts stems from the advantages offered by conceptually simple systems such as lattice gasses or other kinetically constrained models ${ }^{7}$ to rationalize the behavior of real materials.

Here we pursue these efforts by means of studies on two materials composed by high-symmetry molecules which interact via van der Waals and electrostatic forces only. Crystals composed by globular shaped molecules are of interest due to their inherent polymorphism. This arises as a consequence of the interplay between their molecular point-group symmetries and the usually high symmetry of their crystal lattices. Such a circumstance leads in some cases to crystals where the lattice structure corresponds to that of the centerof-mass of molecules sitting at the lattice nodes, this now understood as a time-averaged configuration. Such molecules can execute fast reorientations under usually weak hindering forces. Motions along different crystal axes are required so that the molecular and crystal symmetries become symmorphic. In other words, the presence of a usually highsymmetry crystal lattice for a material composed of molecules with point-group symmetries lower than the lattice space group, forces molecular rotations to take place along different axes so that the lattice symmetry arises as a timeaverage taken over whole periods of molecular rotations. Methylchloromethanes $\left[\left(\mathrm{CH}_{3}\right)_{4-n} \mathrm{CCl}_{n}, \mathrm{Cl}_{n}\right.$ for short $]$ consist on a central carbon atom, and an increasing number of chlorine atoms $(n)$ with a concomitant decrease of methyl groups $(4-n)$. Such a family of compounds retains a marked similitude of its structural properties since the overall molecular symmetry is mostly preserved with increasing $\mathrm{Cl}$ substitution due to the close Van der Waals radii of a chlorine atom and a methyl group. The substitution, however, leads to significant differences in their potential energy landscapes resulting in different polymorphic sequence.

The compound of the series here studied referred to as $\mathrm{Cl}_{4}$ shows one metastable OD phase, with a monotropy relationship to the stable phase. ${ }^{8-11}$ This means that when this compound is cooled down from the liquid phase $(L)$, a first phase transition into a metastable phase (Ia) takes place, and on further cooling a subsequent transition to the stable phase (Ib) occurs. Heating from such a state leads to melting of the stable $\mathrm{Ib}$ phase without subsequent transformation into the metastable Ia orientationally disordered phase. In other words, reaching the Ia state requires freezing from the liquid. The metastable phase Ia shows a fcc structure while stable OD phase Ib displays a rhombohedral lattice. In addition, a low-temperature phase transition from phase Ib leads to $\mathrm{C} 2 / \mathrm{c}$ monoclinic structure. On the other hand all the phase transitions involved in the $\mathrm{Cl}_{1}$ compound are reversible. 
More into specifics, such a compound named tert-butyl chloride, has a low-temperature monoclinic phase with $P 2_{1} / \mathrm{m}$ symmetry which upon heating transforms into tetragonal $P 4 / \mathrm{nmm}^{10,12}$ In this last phase molecules are placed in such a way that the $\mathrm{C}-\mathrm{Cl}$ molecular axis lies along the crystal $c$ axis. Dynamically, this latter phase, ${ }^{13}$ shows molecules rotating about the $\mathrm{C}-\mathrm{Cl}$ axis, and a low amplitude libration corresponding to angular excursions from such a direction. The phase attained upon freezing the normal liquid shows an orientationally disordered character with fcc symmetry. In turn, the compound for $n=4$, carbon tetrachloride, shows a $C 2 / c$ monoclinic low temperature phase and two OD phases. The phase attained while cooling down the liquid again shows a fcc structure and, on further cooling one arrives to the rhombohedral stable OD phase.

Here we report on neutron diffraction results carried out on both liquid and rotationally disordered crystal states of both compounds. Our aim is thus to track down the origin of orientational correlations within the liquid state using the OD fcc crystal as reference states. The important aspect here stems from previous evidences that have shown how shortrange order (SRO) within crystalline phases is kept in the disordered phases of these compounds ${ }^{10,14}$ and therefore the question of whether such SRO still is preserved upon melting naturally arises.

\section{EXPERIMENTS AND DATA ANALYSIS}

\section{A. Experimental}

Materials were used without further purification. The deuterium isotopic purity of the $\mathrm{Cl}_{1}$ compound was better than 99\%. Neutron-diffraction experiments were carried out using the D1B diffractometer at the Institut Laue-Langevin (Grenoble), using a wavelength of $\lambda=1.2805 \AA^{-1}$, as determined by calibration using an alumina sample. The cell used to perform the experiments was a standard vanadium can with a diameter of $9.5 \mathrm{~mm}$ and a wall of $0.1 \mathrm{~mm}$. In order to perform the necessary corrections we have also measured an empty cell and a Cadmium probe. The preparation of the samples in their liquid or disordered crystal phases was carried out in situ, by means of mild thermal treatments. Measurements were performed, on both compounds, at temperatures of 298 and $240 \mathrm{~K}$ corresponding to liquid and crystal phases of $\mathrm{CCl}_{4}$ and at 298 and $238 \mathrm{~K}$ for those pertaining $\left(\mathrm{CD}_{3}\right)_{3} \mathrm{CCl}$, respectively. Data were corrected for absorption, extinction and multiple scattering using the computer code "correct" provided by the Studsvik Neutron Research Laboratory. ${ }^{15}$ Placezk corrections were applied to the corrected data fitting a polynomial function, having $Q^{2}$ and $Q^{4}$ terms, to the obtained spectra. The maximum momentum transfer obtained in this experiments was of $8 \AA^{-1}$, large enough to obtain information about short-range order in the disordered phases using the RMC method, since within such phases intra molecular and intermolecular distances are clearly distinguishable as shown by Jóvári. ${ }^{16}$ Because of the somewhat limited $Q$ range used, special precautions had to be taken to avoid strong truncation ripples in the fourier transformed spectra. To alleviate such a problem recourse was made to previous work on fourier inversion maximum entropy methods ${ }^{17}$ that are specially suited to deal with this kind of problems.

Diffraction patterns for $\mathrm{CCl}_{4}$ within the rotationally disorder crystal show an intense Bragg peak corresponding to the [200] reflection at momentum transfers $Q \approx 1.508 \AA^{-1}$ that corresponds within the melt to those characteristic of the first-sharp-diffraction peak (FSDP). Similar remarks apply to $\left(\mathrm{CD}_{3}\right)_{3} \mathrm{CCl}$. Both disordered crystals having $Z=4$ molecules show fairly close values for the lattice parameters that become $a=8.335 \AA$ and $a=8.669 \AA$, respectively.

\section{B. RMC modeling}

To help with the analysis of experimental data, reverse Monte Carlo simulations ${ }^{18}$ (RMC) fits to the obtained spectra have been performed. The method uses a standard Monte Carlo algorithm to move particles within a simulation box, but instead of minimizing the energy, it is the statistic $\chi^{2}$, calculated from difference between experimental structure factor and the structure factor, calculated from the computer configuration, what is actually minimized. The output consists in 3D molecular configurations that are consistent with experimental data. Here we report on some RMC results for liquid and fcc phases of both compounds. Simulations were carried on using several initial configurations. Bond-angle and bond-length constraints were used since flexible molecules are used to mimic thermal deformations. The closest atom-atom distances of approach were determined from experimental results, and the program GHEMICAL (Ref. 19) was used to determine suitable molecular structures. Estimates of vibration amplitudes were also taken from Refs. 20 and 22.

\section{Simulation of the liquid phases}

The initial configurations of liquid $\mathrm{CCl}_{4}$ and $\mathrm{C}\left(\mathrm{CD}_{3}\right)_{3} \mathrm{Cl}$ were performed within boxes of length 54.34 and $55.8 \AA$, respectively, containing 1000 molecules, which were chosen to correspond to liquid densities under SVP conditions. Minimum distances of approach were set as $3.0 \AA$ for $\mathrm{Cl}_{1}$ and $3.3 \AA$ for $\mathrm{Cl}_{4}$. Once the initial configuration of the molecular center-of-mass was specified, initial random orientations of the molecules were generated. This was verified by calculation of several orientational correlators which show the same random average values for separation distances between molecules of $5.25 \AA<d<12 \AA$. Simulations were carried in all cases until convergence of the $\chi^{2}$ parameter was achieved. Some representative results for both liquids are shown in Figs. 1 and 2. As can be seen good agreement between calculated and experimental spectra was achieved. In addition, a further test of the relevance of the finally obtained configurations was provided by comparisons between our calculated partial pair correlation functions for one of the liquids $\left(\mathrm{Cl}_{4}\right)$ and those derived from previous MD simulations. ${ }^{22,23}$ They were found to agree with a maximum difference of $\simeq 0.2$.

\section{Simulation of the fcc phases}

Diffuse scattering is the intensity that is scattered by disordered crystals and usually appears beneath Bragg reflections. Thermal or static disorder either of substitutional, po- 


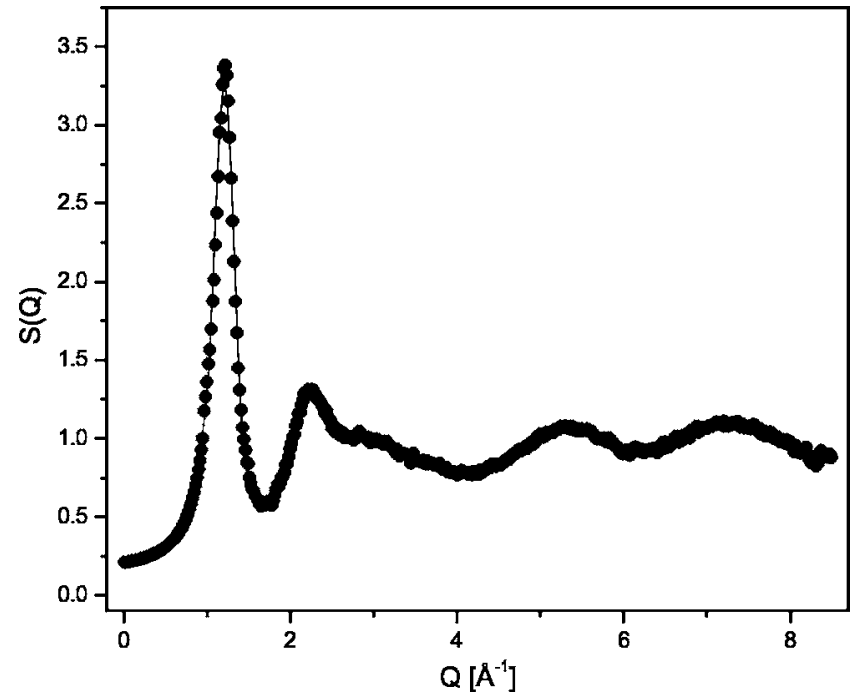

FIG. 1. Comparison of experimental (solid circles) $S(Q)$ structure factor of liquid $\mathrm{C}\left(\mathrm{CD}_{3}\right)_{3} \mathrm{Cl}$ with its $\mathrm{RMC}$ fit (solid line).

sitional, or orientational origin gives rise to such scattering patterns. Furthermore, in the case of orientationally disordered crystal phases such diffuse spectrum contains valuable information about the short-range order present in these phases. Here we will follow steps similar to those employed in the pioneering work of Dolling et al. on the plastic phases of $\mathrm{SF}_{6}$ and $\mathrm{CBr}_{4}$. The static structure factor $S(\mathbf{Q})$, for a disordered polycrystal, can be represented by a sum of Bragg and diffuse components ${ }^{24,25}$

$$
S(Q)=S_{\text {Bragg }}(Q)+S_{\text {diff }}(Q) .
$$

The $S_{\text {Bragg }}(Q)$ term contains information about the timeaveraged crystal structure and is defined by ${ }^{24}$

$$
S_{\text {Bragg }}(Q)=\left|\left\langle b_{Q}\right\rangle\right|^{2},
$$

where $\langle\cdots\rangle$ denotes a thermodynamic average and

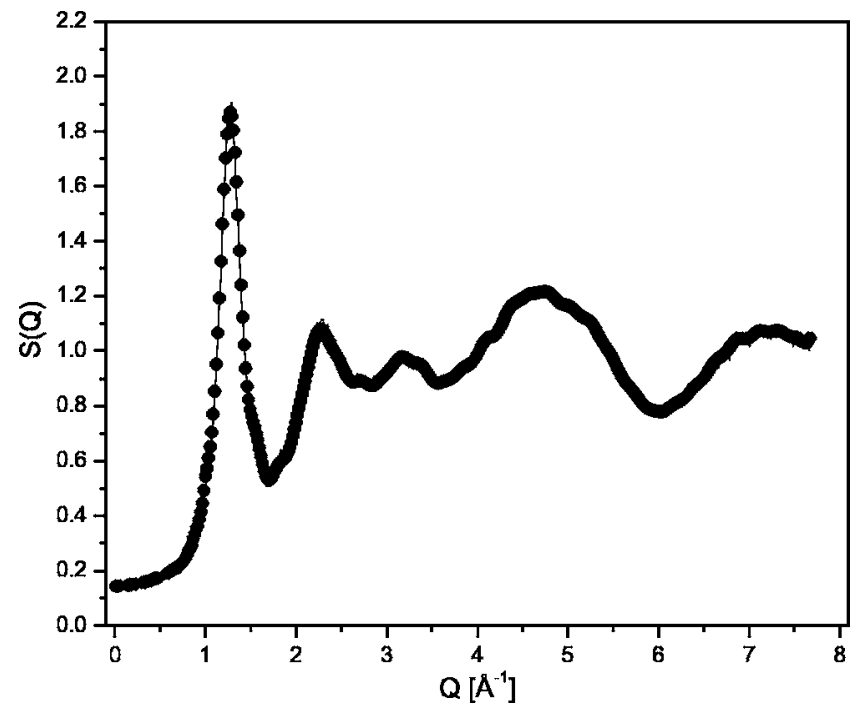

FIG. 2. Reverse Monte Carlo fit (solid line) to the experimental structure factor (solid circles) corresponding to liquid $\mathrm{CCl}_{4}$.
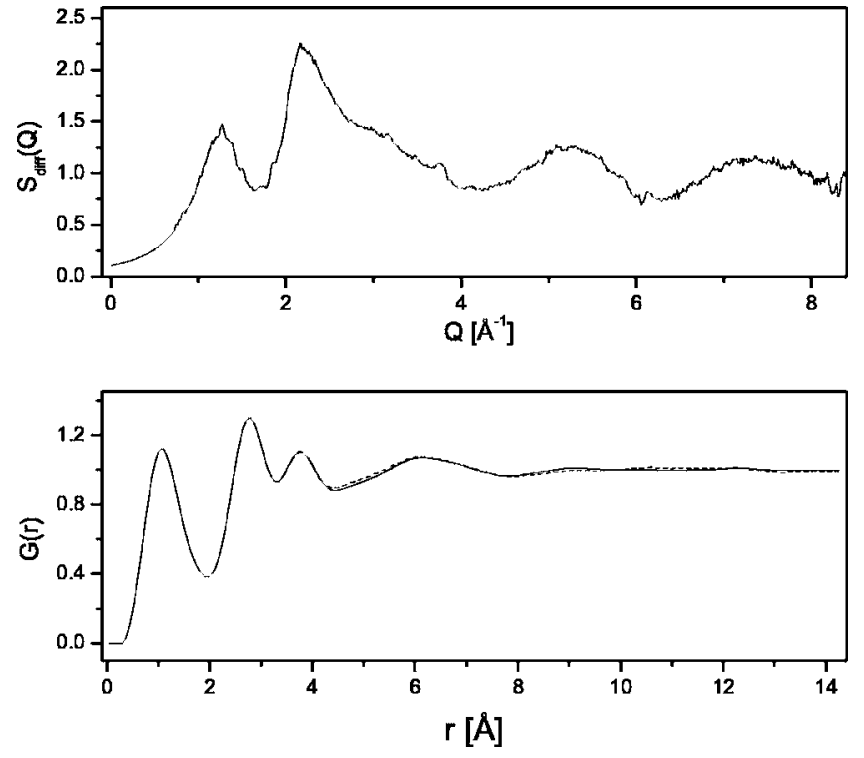

FIG. 3. Experimental diffuse structure factor $S_{\text {diff }}(Q)$ and the corresponding real-space distribution function $G(r)$ (solid line) of $\mathrm{C}\left(\mathrm{CD}_{3}\right)_{3} \mathrm{Cl}$ fcc phase; the dashed line corresponds to the RMC fit.

$$
b_{\mathbf{Q}}=\sum_{j} b_{j} \exp \left(i \mathbf{Q} \cdot \mathbf{r}_{j}\right)
$$

Here $b_{j}$ is the scattering length and $\mathbf{r}_{j}$ the position of the $j$ th nucleus and the sum runs over all the nuclei in the system.

$S_{\text {diff }}(Q)$ arises from the equilibrium fluctuations in $b_{\mathbf{Q}}$ and represents diffuse scattering:

$$
S_{\text {diff }}(Q)=\left\langle\left|\delta b_{Q}\right|^{2}\right\rangle \text {. }
$$

To carry out RMC analysis of the diffuse scattering patterns the starting configurations were generated with molecular centers-of-mass located at the fcc equilibrium positions and random orientations of molecules. This is done in order to alleviate the finite-size problems posed to the RMC method by the simultaneous presence of both Bragg and diffuse-scattering peaks (see Ref. 26). The number of molecules employed were of 864 molecules for both $\mathrm{Cl}_{4}$ and $\mathrm{Cl}_{1}$ corresponding to simulation boxes of 50 and $52 \AA$, respectively. Such length comprises $6 \times 6 \times 6$ cells. The same tests as in the liquid phase were performed, ensuring a complete random initial orientation of molecules.

An alternative route such as that described in Ref. 27 was also followed to ensure the reliability of the obtained results. It is based upon RMC modeling using the experimentally derived $G(r)$ rather than the static structure factor. The former was obtained by transforming $S_{\text {diff }}(Q)$ into real space using methods referred to above. The RMC simulations for the crystals were then performed keeping the molecule centers-of-mass in their fcc lattice points.

In Figs. 3 and 4 the agreement between calculated and experimental spectra can be gauged. 

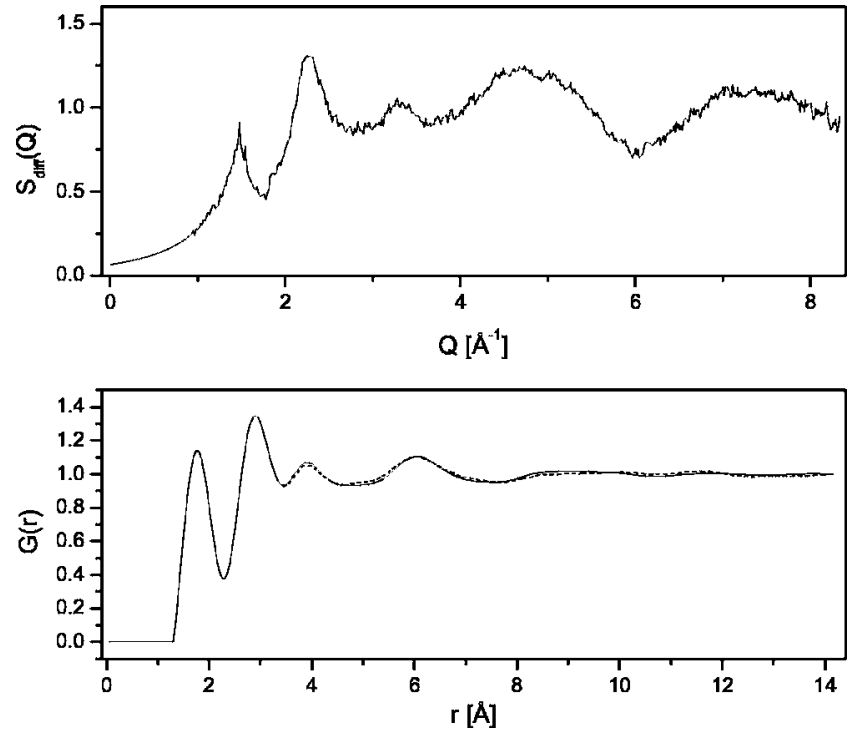

FIG. 4. Upper frame shows experimental $S_{\text {diff }}(Q)$, the lower displays $G(r)$ (solid line) compared with RMC fit (dashed line); for $\mathrm{CCl}_{4}$ fcc phase.

\section{RESULTS}

\section{A. Carbon tetrachloride}

\section{Liquid phase}

The nature and extent of orientational short-range-order in liquid $\mathrm{CCl}_{4}$ has been a topic many times visited since early reports, ${ }^{20,23,28}$ up to very recent times. ${ }^{29}$ The Apollo model for a $\mathrm{Cl}_{4}$ molecular dimer first proposed by Egelstaff et al., ${ }^{28}$ in which the vector defined by the $\mathrm{C}-\mathrm{Cl}$ bond of two molecules is parallel and lies in the same axis (configuration labeled as A in Fig. 5), seems to explain X-ray and neutrondiffraction spectra for the liquid. ${ }^{22,23}$ Pictorially, this configuration shows one of the chlorine atoms placed within the
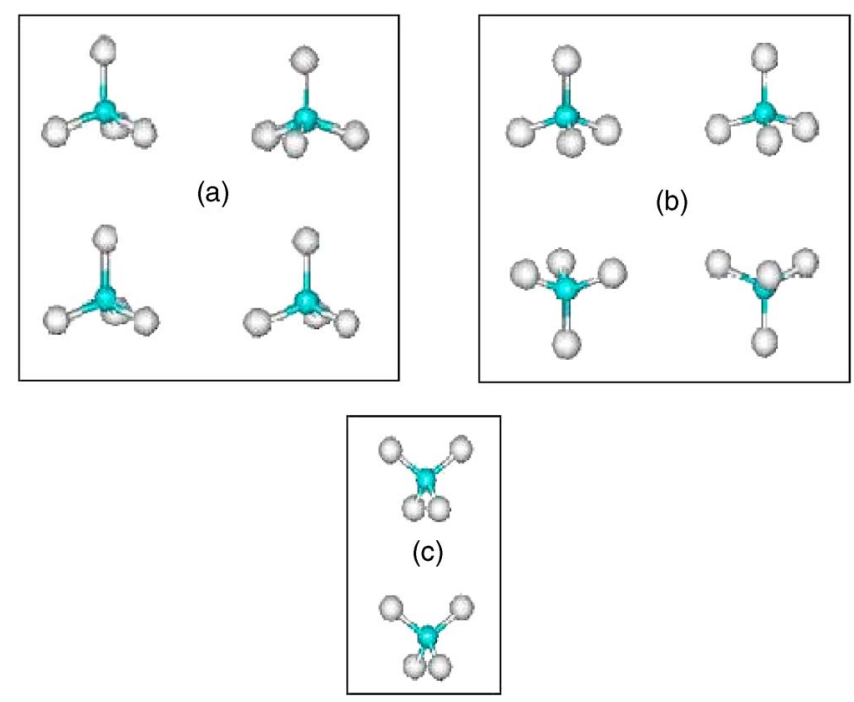

FIG. 5. (Color online). Orientational models describing the nearest-neighbor arrangement. (a) Apollo type. (b) Face-to-face configuration. (c) Interlocked arrangement.

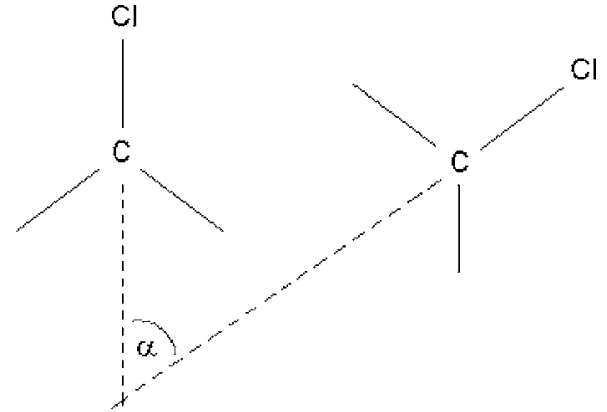

FIG. 6. Definition of the $\alpha$ angle, defined by vectors $\mathbf{r}_{\mathrm{CCl}}$ of different molecules.

hollow formed by the three chlorine atoms (sometimes called the "face" of the molecule) of the adjacent molecule. A slight variation of this model leads to the interlocked configuration where molecule "edges" formed by two chlorine atoms are perpendicular to those of a neighbor molecule, allowing an interpenetration of both tetrahedra, a geometry also depicted in Fig. 5(c). Another sort of dimer arrangement is the "face to face" type configuration, where the vector defined by the $\mathrm{C}-\mathrm{Cl}$ bond is antiparallel, Fig. 5(b), lying on the same axis as in the crystalline state, ${ }^{30}$ or being slightly displaced as in the configuration proposed by Lowden et al. ${ }^{31}$ (in what follows Lowden-Chandler interlocked configuration).

To provide a quantitative measure of orientational shortrange order we have chosen to study the relative orientation and position of nearest molecules in terms of the distribution $\mathbf{P}$ of values of the angle $\alpha$ defined by vectors $\mathbf{r}_{\mathrm{CCl}}$ of different molecules (Fig. 6), having each molecule four equivalent $\mathbf{r}_{\mathrm{CCl}}$ vectors. Such distributions were calculated for pairs of molecules within distances of $d<5.25 \AA$, that correspond to nearest-neighbor separation. The distributions thus comprise an average taken over the 16 possible $\alpha$ angles and the result is shown in Fig. 7. Data displayed in Fig. 7 thus include all possible effects due to competition between different favorable orientations. The procedure was repeated for increasing distances of separation. The distribution, as expected, became more featureless as the distance increases, attaining values nearly uniformly distributed for distances above some $10 \AA$. From the graph shown in Fig. 7 we infer that neither Apollo-type or interlocked orientations are truly dominant over others since peaks at $\cos (\alpha)=1$ and $\cos (\alpha)=-0.33$ would have to be more relevant otherwise. On the other hand, face to face type orientations are more abundant since these lead to contributions for $\cos (\alpha)=-1$, which indeed are present in the figure. Finally, even face to face or LowdenChandler interlocked molecular arrangements are compatible with our data, leading both to contributions not far from $\cos (\alpha) \simeq 0.33$, the deviation towards higher values in $\cos (\alpha)$ in our data, is probably a signature of lesser defined orientational correlations than those derived from the somewhat idealized models here discussed.

An estimate of the strength of orientational correlations and how these translate into features displayed by the radial distributions is provided by data shown in Fig. 8. There, the resulting distributions from a fully relaxed RMC simulation are compared to results corresponding to complete random- 

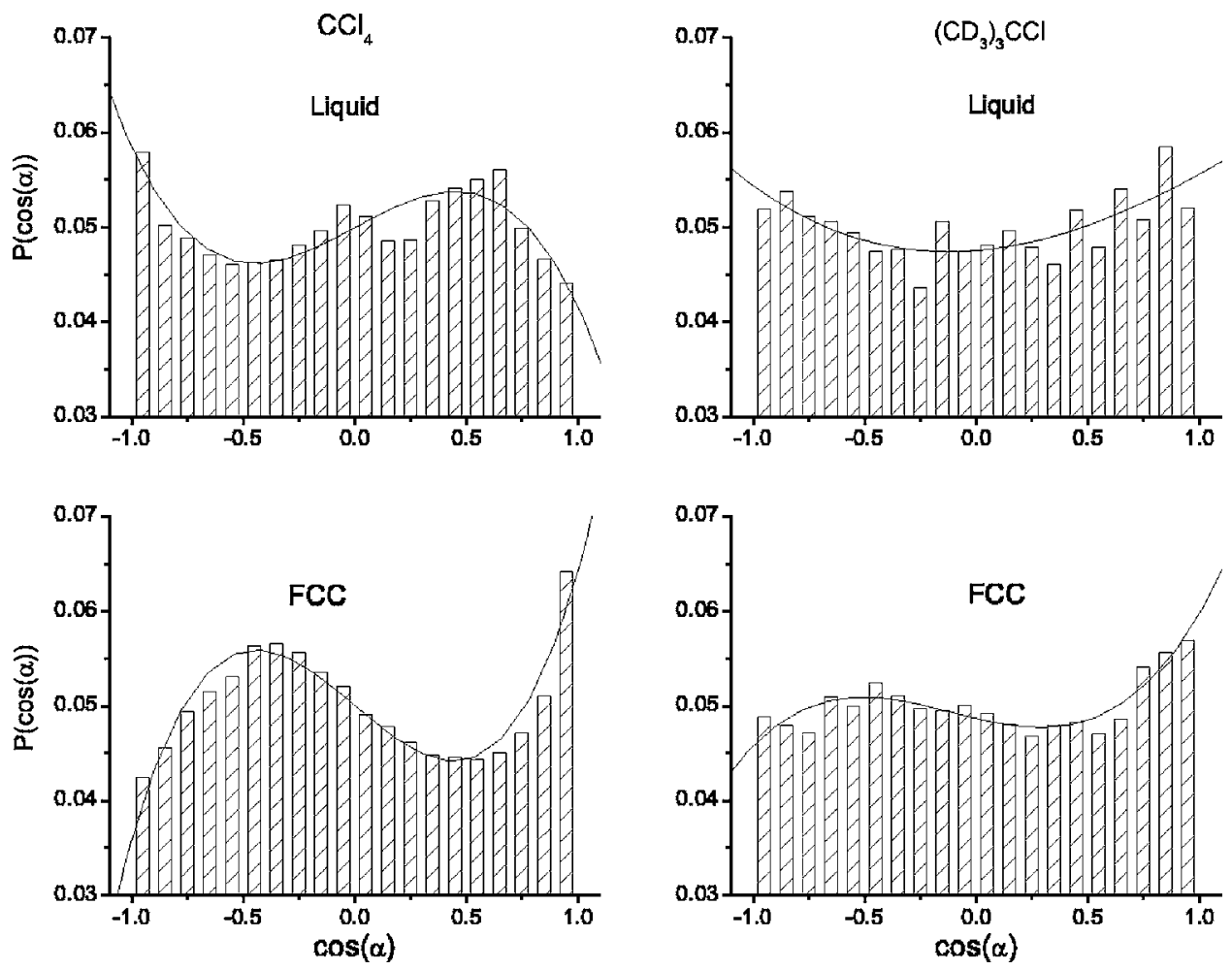

FIG. 7. Cosine distribution of $\alpha$ angles for $\mathrm{CCl}_{4}$ and $\left(\mathrm{CD}_{3}\right)_{3} \mathrm{CCl}$ compounds, with $d<5.25 \AA$ and $d<7 \AA$ for liquid and fcc phases, respectively. Solid lines are just guides to the eye. ization of molecular orientations. The optimal $G(r)$ functions displayed in the figure just referred to show far marked structure than those corresponding to random orientations. The comparison of random and fully relaxed distributions thus lends further support to the soundness of angular distributions displayed in Fig. 7.

A less restricted view of the short-range-order is provided by a representation of the three-dimensional structure as a projection of the coordinates of molecular centers into a ref-

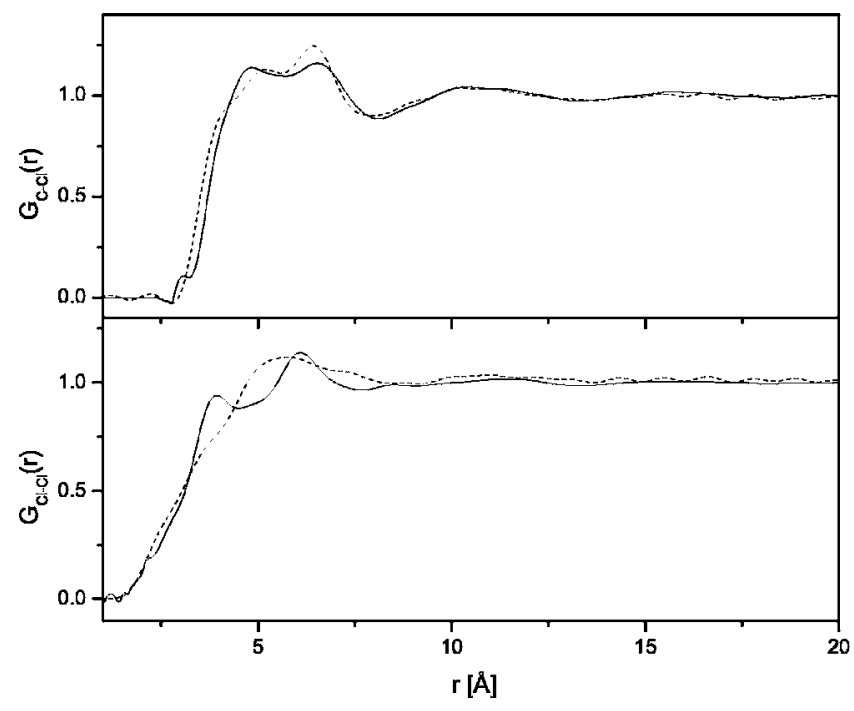

FIG. 8. A comparison of the partial radial distribution functions for $\mathrm{C}-\mathrm{Cl}$ and $\mathrm{Cl}-\mathrm{Cl}$ resulting from an optimized $\mathrm{RMC}$ fit (solid lines) of data for liquid $\mathrm{CCl}_{4}$ compared to those corresponding to a configuration with complete random molecular orientations (dashes). erence system fixed on a central molecule. The $\mathrm{C}$ atom of the central molecule is placed at the origin, one of its $\mathbf{r}_{\mathrm{CCl}} \mathrm{vec}-$ tors defines the $Z$ axis, being the $Z$ coordinate of this $\mathrm{Cl}$ atom positive. The $X Z$ plane is specified by an additional $\mathbf{r}_{\mathrm{CCl}}$ vector, being the $X$ coordinate of this second $\mathrm{Cl}$ atom positive. In these intensity contours, vectors were normalized to unity, since we are interested only in angles and not in the distances involved. The distribution of molecules at distances less than $5.25 \AA$ (corresponding to nearest neighbors) is such that molecules tend to be localized opposite to $\mathrm{Cl}$ atoms Color plots of the relevant correlations are given as suppplementary material, ${ }^{21}$ which provides a further evidence that molecule dimers have a face-to-face or Lowden-Chandler interlocked conformation, a configuration supported by the results of Rey et al. ${ }^{22}$ Finally our results lend additional support to findings reported in Ref. 32 where a more primitive molecular model was employed in the RMC simulations.

\section{The fcc phase}

The short-range order within the rotator-phase crystal is determined following the same steps taken for the liquid phase, by calculation of angular and positional correlations between neighboring molecules. The value of the lattice parameter for the fcc phase at a temperature $T=240 \mathrm{~K}$ (Ref. 10) $(a=8.335 \AA)$ yields first- and second-neighbor distances of $d \approx 5.9 \AA$ and $d=a$, respectively, account made of the simulation box size. In Fig. 9 the $\cos (\alpha)$ distribution function corresponding to correlations between first $(d<7 \AA)$ and second neighbors $(7<d<9 \AA)$ is shown. Astonishingly, it can be seen that a significant difference between angle correlations exists between results obtained for such two distances. For $7<d<9 \AA$ corresponding to second neighbors, the angular distribution behaves much alike the liquid phase, 

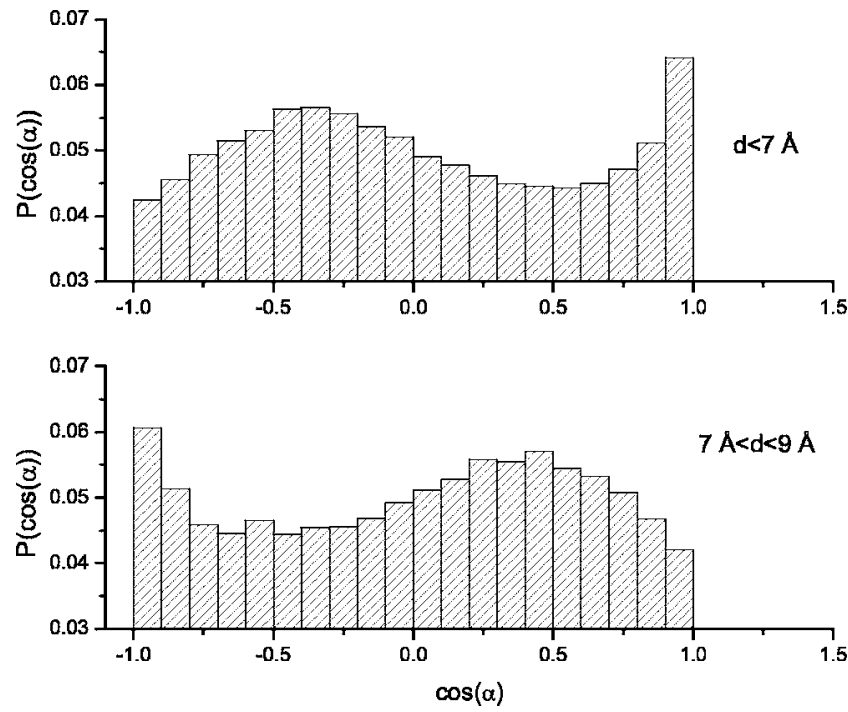

FIG. 9. Angular correlation for $\mathrm{CCl}_{4}$ fcc phase. Distances $d<7 \AA$ and $7<d<9 \AA$ corresponding to first and second neighbors from reference molecule.

whereas for $d<7 \AA$ it is suggestive of a different kind of short-range order. As written above, an Apollo configuration would result in maxima of the cosine distribution function for $\cos (\alpha)=1$ and $\cos (\alpha)=-0.33$, as indeed are here observed.

It is also striking to compare angular correlations of this OD phase to those of the liquid. From Fig. 7 we see a rather different ordering pattern for both phases. While the liquid shows a dominance of face-to-face configurations, the Apollo configuration seems to better describe relative orientation of molecules within the fcc phase. This means that transit from liquid to the fcc phase involves a rotation of first neighbors of $71^{\circ}$ as well as a concomitant center-of-mass displacement.

A representation of the three-dimensional structure projections, corresponding to first neighbors is shown in supplementary material. ${ }^{21}$ In this case, at variance with the liquid, molecules tend to concentrate in the positive $Z$ direction. Such a distribution again corroborates the Apollo-type configuration as the most probable to describe short-range order within this phase.

\section{B. Tert-butyl-chloride}

\section{Liquid phase}

Unlike the nonpolar $\mathrm{CCl}_{4}$ case, the $\mathrm{C}\left(\mathrm{CD}_{3}\right)_{3} \mathrm{Cl}$ molecule has a privileged direction defined by the $\mathbf{r}_{\mathrm{CCl}}$ vector which follows that of the molecule dipole moment. This implies that there exists only one $\alpha$ angle belonging to two different molecules separated by a distance $d$. In Fig. 7 this angular correlation is depicted. The statistics of data shown in the figure just referred to are poorer than those previously shown since averages are now taken over a single $\mathrm{C}-\mathrm{Cl}$ bond direction per molecule, and this could not be compensated by taking averages over more configurations. At any rate, the figure shows that the preferred $\mathrm{C}-\mathrm{Cl}$ relative orientations
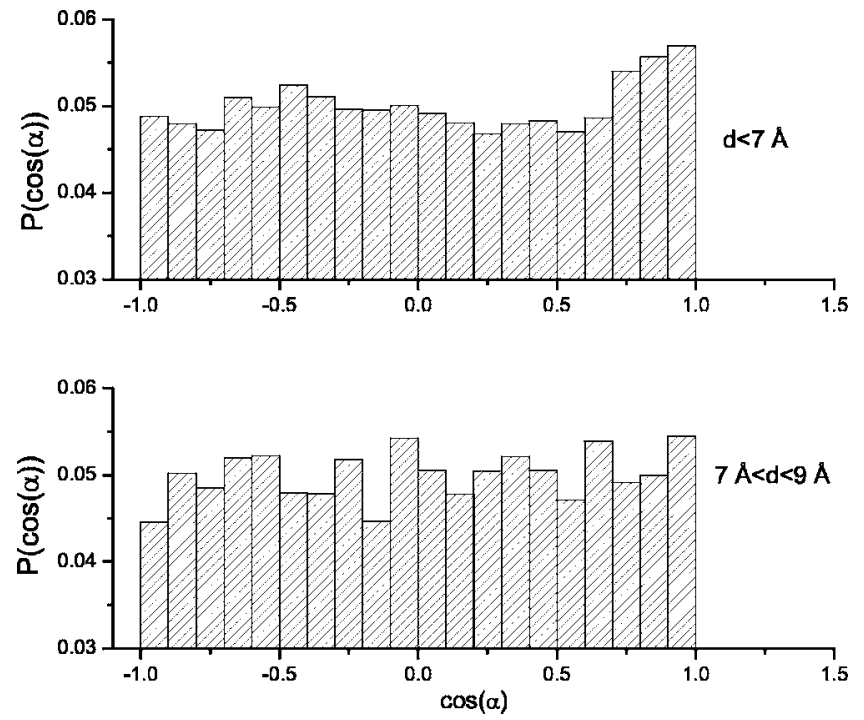

FIG. 10. C- $\mathrm{Cl}$ angle correlation function corresponding to fcc phase of $\mathrm{C}\left(\mathrm{CD}_{3}\right)_{3} \mathrm{Cl}$ compound.

correspond to $\cos \alpha \simeq 1$ and $\cos \alpha \simeq-1$ unlike in the $\mathrm{Cl}_{4}$ case: that is, no "pure" configuration can be assigned to such an angle distribution, Apollo and face-to-face configurations are in competition.

A clue to understand the origin of such a behavior can be found taking into account the dipole moment of the molecule $(2.16 \mathrm{D})$ that tends to orient molecules ferroelectrically. Therefore this angular correlation results from a compromise between the electrostatic force that tends to orient the molecules in an Apollo configuration, and the steric (dispersion force) effects making the molecules to approach as much as possible, as in the face-to-face configuration.

Concerning the projection of the central carbon positions, a similar spatial distribution to that of the $\mathrm{Cl}_{4}$ compound is found (an additional figure is given in Ref. 21), which is compatible with the face-to-face configurations of dimers. Concerning the absence of the bright spot in the $Z$ direction related to the Apollo configuration, it only can be explained if the occurrence of such relative orientation is rather poor.

\section{The fcc phase}

The details concerning the fcc phase are shown in Fig. 7 and Ref. 33 which display the orientational and positional features as done for the previous compound. Both figures evidence that the preferred relative orientation of a dimer is now that of an Apollo configuration. In the fcc phase no contribution to $\cos \alpha \simeq-1$ is found, that is to say, no face to face configuration contributes to the short-range order.

It is worth remarking here that important differences in short-range -order between $\mathrm{Cl}_{4}$ and $\mathrm{Cl}_{1}$ may hold the key to explain the different polymorphism of both compounds. Unlike the case of $\mathrm{Cl}_{4}$, where second neighbors have face-toface relative orientation, $\mathrm{Cl}_{1}$ shows for such neighbors dominantly Apollo configurations (Fig. 10), that is the relative orientations at short range correspond to a ferroelectric arrangement. Such alignment of molecular dipoles is, however, short ranged and does not last beyond the next-nearest- 
neighbor shell. This arrangement is reminiscent of that appearing within the tetragonal $P 4 / \mathrm{nmm}$ phase that forms below $217.9 \mathrm{~K}$ which shows ferroelectrically aligned chains of molecules along the crystal $c$ direction, while the direction of the dipole moment along such chains is reversed in the crystal $a-b$ directions. The fact that such local order is absent in the OD fcc phase of $\mathrm{Cl}_{4}$ nicely unveils the action of electric dipole forces. In this latter case, electrostatic forces are generated by a sizable induced-dipole contribution [average value estimated as $0.26 \mathrm{D}$ (Ref. 33)] plus a that generated by a small molecular octupole moment $\left(15 \pm 3 \mathrm{D} \AA^{2}\right) .^{33}$

\section{OUTLOOK AND CONCLUSIONS}

In this work we analyzed orientational correlations at short range in liquid and plastic phases of $\mathrm{CCl}_{4}$ and $\mathrm{C}\left(\mathrm{CD}_{3}\right)_{3} \mathrm{Cl}$ by means of the combined used of neutrondiffraction and RMC modeling. As expected, the obtained results for liquid $\mathrm{Cl}_{4}$ agree with previous determinations, ${ }^{20,23,30,31}$ that ensures the reliability of the procedures here followed. The basic picture within such liquid portrays neighboring molecules ordered mostly in terms of face-to-face and Lowden-Chandler arrangements. The important result here concerns the rather different orientational arrangements found for the fcc plastic phase. There, both angular correlations and nearest neighbors distributions are significantly different from those found for the liquid. Such a different behavior is understood on the basis of the energetics of a pair of $\mathrm{Cl}_{4}$ molecules separated by a distance given by the maximum of the $G_{\mathrm{CC}}(r)$ partial pair correlation function. This shows that the arrangements with increasing stability are interlocked molecules, Apollo model, face-to-face; and therefore one expects that in the absence of long-range effects brought forward by the presence of the periodic lattice, nearest-neighbor molecules will adopt the minimum energy configuration.

The results concerning $\mathrm{Cl}_{1}$ also merit some remarks. Within the liquid, and contrary to simple assumptions leading to the expectancy of dominant configurations involving the alignment (or counteralignment) of molecular dipoles, one finds in the angle distributions only subtle features indicating some predominance of ferro and antiferroelectric arrangements. These correlations are largely enhanced within the disordered crystal phase, thus favoring ferroelectric arrangement at short distances. Again, and contrary to simple expectancies, such well defined correlations fade away for distances comparable to the second neighbors shell.

Finally, Fig. 11 shows a comparison between the liquid radial distribution functions and $G_{\text {orient }}(r)$ that is related to the Fourier transform of $S_{\text {diff }}(Q)$. The distances are scaled by the average molecular radii $(\sigma)$. The estimated values for the molecular diameters are $2 \sigma=7 \AA$ for $\mathrm{Cl}_{4}$ and $2 \sigma=7.04 \AA$ for $\mathrm{Cl}_{1} \cdot{ }^{22}$ The first nontrivial result inferred from a glance to such graphs concerns the rather different spatial extents where $G_{\text {orient }}(r)$ shows noticeable oscillatory structure. The nonpolar (but polarizable) $\mathrm{Cl}_{4}$ shows such features up to about $5.5 \sigma$ while that for $\mathrm{Cl}_{1}$ vanishes for distances in excess

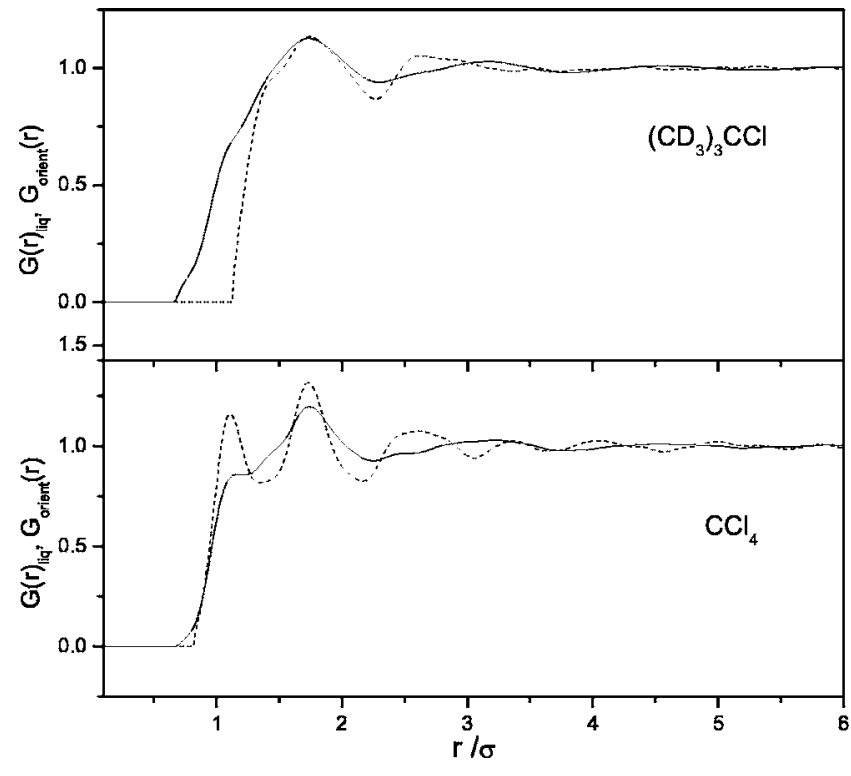

FIG. 11. Upper frame displays $G_{\text {liq }}(r)$ (solid line) and $G_{\text {orient }}(r)$ (dashed line) for $\mathrm{C}\left(\mathrm{CD}_{3}\right)_{3} \mathrm{Cl}$ compound. Lower frame shows the corresponding functions for $\mathrm{CCl}_{4}$.

of $4.5 \sigma$. This difference in behavior can tentatively be ascribed to frustration effects present in the latter sample due to competing ferromagnetic and antiferroelectric alignment of molecular dipoles. Such effects are obviously absent in the former crystal which can only experience weaker induceddipole interactions which are about one order of magnitude weaker than those generated by permanent dipoles in $\mathrm{Cl}_{1}$. Also worth noticing from Fig. 11 is the far sharply defined shapes of both the liquid $G(r)$ and $G_{\text {orient }}(r)$ for $\mathrm{Cl}_{4}$ if compared to those for $\mathrm{Cl}_{1}$. In particular, the effects of the marked disorder in orientation of nearest neighbors within the liquid and the alignment of molecular dipoles for crystal $\mathrm{Cl}_{1}$ translates here into very different shapes of the radial distributions for distances comparable to those of molecular contacts. In fact, the rise up of the radial distribution for $\mathrm{Cl}_{4}$ at distances close to $\sigma=1$ is far steeper than that of liquid $\mathrm{Cl}_{1}$. This comes as a consequence of the spatial correlations between $\mathrm{CD}_{3}$ groups which involve a wider range of distances than those of $\mathrm{Cl}-\mathrm{Cl}$ contacts. Once a better defined orientational local order sets in within the OD crystal, intermolecular correlations become better defined, making $G_{\text {orient }}(r)$ closer to that of $\mathrm{Cl}_{4}$.

In summary, we have carried out a quantitative comparison of the extent of orientational short-range order in two materials amanable to be studied within their molten states as well as within the high-symmetry rotator-phase crystals. The latter, which are to be considered as having a thermodynamic stability closer to the liquid than to that of the fully ordered states show a dramatic enhancement of angular correlations with respect to those of the liquids. Finally, our results serve to qualify usual assumptions concerning the strength of orientational correlations in polar liquids. In fact, the study of the polar $\mathrm{C}\left(\mathrm{CD}_{3}\right)_{3} \mathrm{Cl}$ compound shows that such correlations within both liquid and OD phases are indeed less marked 
than those of the nonpolar, but highly polarizable $\mathrm{CCl}_{4}$. This final result emphasizes the role played by higher-order interactions involving molecular polarization in governing particle arrangements at short distances. Their importance is known from hyper-Raman scattering experiment, ${ }^{34}$ although explicit means to model them are only starting to be available. $^{35}$

\section{ACKNOWLEDGMENTS}

This work was supported by the Spanish Ministry of Science and Technology (Grant Nos. BFM2002-01425, MAT2002-04540-C05-03) and by the catalonian goverment (Grant No. SGR2002-00152). We would thank to R. Rey for helpful discussions.
${ }^{1}$ D. L. Price, M. L. Saboungi, and F. J. Bermejo, Rep. Prog. Phys. 66, 407 (2003).

${ }^{2}$ A. Criado, M. Jimenez-Ruiz, C. Cabrillo, F. J. Bermejo, R. Fernandez-Perea, H. E. Fischer, and F. R. Trouw, Phys. Rev. B 61, 12082 (2000); M. Jimenez-Ruiz, M. A. Gonzalez, F. J. Bermejo, M. A. Miller, N. O. Birge, I. Cendoya, and A. Alegria, ibid. 59, 9155 (1999).

${ }^{3}$ B. Bagchi and A. Chandra, Phys. Rev. Lett. 64, 455 (1990); T. Grochulski, L. Pszczolkowski, and M. Kempka, ibid. 68, 3635 (1992).

${ }^{4}$ See, for instance, G. Evrard and L. Pusztai, J. Phys.: Condens. Matter 17, S37 (2005); M. Reicherst and H. Stark, Phys. Rev. E 69, 031407 (2004); J. P. Rino and N. Studart, Phys. Rev. B 59, 6643 (1999); D. L. Price, M. L. Sabougni, Y. S. Badyal, J. Wang, S. C. Moss, and R. L. Leheny, ibid. 57, 10496 (1998); S. Lavy, P. Launois, and R. Moret, ibid. 53, R10532 (1996); J. Dawidowski, A. Chahid, F. J. Bermejo, E. Enciso, and N. G. Almarza, Phys. Rev. E 52, 2787 (1995).

${ }^{5}$ K. R. Purdy, Z. Dogic, S. Fraden, A. Rühm, L. Lurio, and S. G. J. Mochrie, Phys. Rev. E 67, 031708 (2003); R. D. Kamien and A. J. Levine, Phys. Rev. Lett. 84, 3109 (2003); M. G. Brereton and T. A. Vilgis, Macromolecules 29, 7588 (1996).

${ }^{6}$ F. Leonard, N. C. Bartelt, and G. L. Kellogg, Phys. Rev. B 71, 045416 (2005); K. Theis-Bröhl, B. P. Toperverg, V. Leiner, A. Westphalen, H. Zabel, J. McCord, K. Rott, and H. Bruckl, ibid. 71, 020403 (2005); A. T. Boothroyd, P. G. Freeman, D. Prabhakaran, A. Hiess, M. Endrele, J. Kulda, and F. Altorfer, Phys. Rev. Lett. 91, 257201 (2003); S. R. Ren and I. W. Hamley, Physica B 322, 110 (2002).

${ }^{7}$ See, for instance, P. Sollich, and F. Ritort, in Proceedings of a workshop on glassy behavior of kinetically constrained models, Preface, edited by Editors, Special Issue of J. Phys.: Condens. Matter14(7) (2002); M. Jimenez-Ruiz, A. Criado, F. J. Bermejo, G. J. Cuello, F. R. Trouw, R. Fernández-Perea, H. Löwen, C. Cabrillo, and H. E. Fischer, Phys. Rev. Lett. 83, 2757 (1999); K. Frobose, F. Bonnemeier, and J. Jäckle, J. Phys. A 29, 485 (1996).

${ }^{8}$ P. Negrier, L. C. Pardo, J. Salud, J. Ll. Tamarit, M. Barrio, D. O. Lopez, A. Wurflinger, and D. Mondieig, Chem. Mater. 14, 1921 (2002).

${ }^{9}$ L. C. Pardo, M. Barrio, J. Ll. Tamarit, D. O. Lopez, J. Salud, P. Negrier, and D. Mondieig, Chem. Phys. Lett. 308, 204 (1999).

${ }^{10}$ J. Ll. Tamarit, D. O. Lopez, X. Alcobé, M. Barrio, J. Salud, and L. C. Pardo, Chem. Mater. 12, 555 (2000).

${ }^{11}$ R. Rudman and B. Post, Science 154, 1009 (1966).

${ }^{12}$ R. Rudman, J. Mol. Struct. 485-486, 281 (1999).

${ }^{13}$ T. Hasebe and S. Ohtani, J. Chem. Soc., Faraday Trans. 84, 187
(1988).

${ }^{14}$ R. Rudman, J. Chem. Phys. 66, 3139 (1977).

${ }^{15}$ Program available in the website http://www.studsvik.uu.se

${ }^{16}$ P. Jóvári, M. György, L. Pusztai, and E. Sváb, J. Chem. Phys. 114, 8082 (2001).

${ }^{17}$ F. J. Bermejo, J. Santoro, and M. Alvarez, Nucl. Instrum. Methods Phys. Res. B 42, 281 (1989); F. J. Bermejo, F. J. Mompean, E. Enciso, and M. Alvarez, ibid. 34, 505 (1988).

${ }^{18}$ For reviews on applications of the method see R. L. McGreevy, J. Phys.: Condens. Matter 13, R877 (2001); T. Arai and R. L. McGreevy, ibid. 17 S23-S30, Special Issue (2005).

${ }^{19}$ Program available at URL http://www.bioinformatics.org/ ghemical/

${ }^{20}$ F. J. Bermejo, E. Enciso, J. Alonso, N. Garcia, and W. S. Howells, Mol. Phys. 64, 1169 (1988).

${ }^{21}$ See EPAPS Document No. E-PRBMDO-72-093525 for supplementary figures. This document can be reached via a direct link in the online article's HTML reference section or via the EPAPS homepage (http://www.aip.org/pubservs/epaps.html).

${ }^{22}$ R. Rey, L. C. Pardo, E. Llanta, K. Ando, D. O. López, J. Ll. Tamarit, and M. Barrio, J. Chem. Phys. 112, 7505 (2000).

${ }^{23}$ A. H. Narten, M. Danford, and H. A. Levy, J. Chem. Phys. 46, 4875 (1967).

${ }^{24}$ G. Dolling, B. M. Powell, and V. F. Sears, Mol. Phys. 37, 1859 (1979).

${ }^{25}$ F. J. Bermejo, A. Criado, R. Fayos, R. Fernández-Perea, H. E. Fischer, E. Suard, A. Guelylah, and J. Zuñiga, Phys. Rev. B 56, 11536 (1997).

${ }^{26}$ D. A. Keen, M. G. Tucker, and M. T. Dove, J. Phys.: Condens. Matter 17, S15 (2005).

${ }^{27}$ L. Karlsson and R. L. McGreevy, Physica B 234-236, 100 (1997).

${ }^{28}$ P. A. Egelstaff, D. I. Page, and J. G. Powles, Mol. Phys. 20, 881 (1971).

${ }^{29}$ L. Temleitner and L. Pusztai J. Phys.: Condens. Matter 17, S47S57Special Issue (2005)

${ }^{30}$ P. Jedlovszky, J. Chem. Phys. 107, 7433 (1997).

${ }^{31}$ L. J. Lowden and D. Chandler, J. Chem. Phys. 61, 5228 (1974).

${ }^{32}$ L. Pusztai and R. L. McGreevy, Mol. Phys. 90, 533 (1997).

${ }^{33}$ Units given in $\mathrm{D}=3.33564 \times 10^{-30} \mathrm{C} \mathrm{m}$. Values taken from $\mathrm{E}$. Llanta and R. Rey, Chem. Phys. Lett. 340, 173 (2001); J. E. Pedersen and S. R. Keiding, IEEE J. Quantum Electron. 28, 2518 (1992).

${ }^{34}$ S. Kinoshita, W. Tsukumari, Y. Shimada, and T. Yagi, J. Opt. Soc. Am. B 10, 1017 (1993).

${ }^{35}$ M. Masia, M. Probst, and R. Rey, J. Chem. Phys. 121, 7362 (2004). 